* Einsatz neuer Lehr-Lernformen an Hochschulen

* Neue Buchreihe

\section{Aktuelles aus der Abteilung Medienpädagogik}

\section{Einsatz neuer Lehr-Lern- formen an Hochschulen mit Hilfe Didaktischer Design Pat- tern}

\section{Rose Vogel \& SVEN WiPper- MANN}

\begin{abstract}
Die rasante Entwicklung im Bereich der Informations- und Kommunikationstechnologien stellt auch die Hochschullehre vor neue Herausforderungen: Wie können digitale Medien didaktisch sinnvoll in den LehrLernkontext integriert werden? Über welche Medienkompetenz muss ich verfügen, um meinen Lehrinhalt mit neuen Medien zu unterstützen? Häufig bleiben diese Fragen unbeantwortet, da einschlägige Konzepte für eine sinnvolle Integration fehlen. Im Verbundprojekt "Virtualisierung im Bildungsbereich (VIB)" wurden unterschiedliche Integrationskonzepte für computergestützte Lehr-Lernformen entwickelt und erprobt. Eine Dokumentation dieser Konzepte fand mit Hilfe Didaktischer D esign Pattern statt, um den Herausforderungen der neuen Medien im Hochschulkontext gerecht zu werden.
\end{abstract}

\section{Einleitung}

Lernen mit neuen Medien, e-Learning, Blended Learning - all diese Begriffe sind in der letzten Zeit in unterschiedlichsten Kontexten zu Modewörtern avanciert. Auch die Medienpädagogik bedient sich dieser Buzzwörter und stellt insbesondere die Mediendidaktik, deren Zielsetzung in der Verbesserung des Lehrens und Lernens in verschiedenen Bildungssituationen liegt, vor neue Herausforderungen. Dieser Prozess soll durch den speziellen Einsatz und die G estaltung didaktischer Medien unterstützt werden (vgl. Reinmann-Rothmeier 2002). Doch häufig fehlen einschlägige Konzepte, um Lehr-Lernsituationen didaktisch sinnvoll mit digitalen Medien zu unterstützen und somit eine Verbesserung des LehrLernprozesses anzuregen. Einer der G ründe dafür liegt sicherlich in der enormen Dynamik im Bereich der Informations- und Kommunikationstechnologien und der für den Einsatz notwendigen Medienkompetenz. Diese stetig neuen bzw. veränderten Möglichkeiten digitaler Medien mit ihren speziellen Anforderungen für Lehrende und Lernende stellen - neben der etwaigen Konfusion - auch eine Herausforderung dar, über neue, differenzierte Lehr-Lernformen nachzudenken, damit die Chancen, digitale Medien mit ihrem didaktischen (und nicht nur technischen!) Mehrwert in unterschiedliche Bildungssituationen $\mathrm{zu}$ integrieren, nicht ungenutzt bleiben.

Das vom Land Baden-Württemberg im Rahmen der Virtuellen Hochschule geförderte Verbundprojekt VIB ${ }^{1}$ hat in den letzten fünf Jahren didaktische Konzepte für den sinnvollen Einsatz digitaler Medien in der Hochschullehre entwickelt und versucht, sich den digitalen Herausforderungen $\mathrm{zu}$ stellen. Diese computerunterstützten Lehr-Lernformen, die innerhalb unterschiedlicher Disziplinen entstanden sind, wurden durch einen entsprechenden Einsatz in Hochschulveranstaltungen für Lehramtsstudierende des Grund- und Hauptstudiums evaluiert und weiterentwickelt. D as Erfahrungswissen, das während der Laufzeit des VIB-Projekts von den wissenschaftlichen Mitarbeiterinnen und Mitarbeitern angeeignet wurde, ist im Sinne eines Wissensmanagement-Prozesses (vgl. ReinmannRothmeier \& Mandl 2000) identifiziert, expliziert, kodifiziert, dokumentiert, kommuniziert und genutzt worden, um es nachhaltig an der Hochschule zu verankern.

\section{Herausforderung der Dokumen- tation}

Die Herausforderung besteht nun darin, dieses "didaktische Expertenwissen" in geeigneter Weise zu erheben und so aufzubereiten, so dass es für Kolleginnen und Kollegen aus anderen Fachdisziplinen nutzbar wird. Dies erfordert eine Form der Beschreibung von Veranstaltungskonzeptionen, die vom konkreten Fach absieht, aber so präzise ist, dass das didaktische Vorgehen von weiteren Lehrpersonen in anderen Inhaltskontexten wiederholt bzw. punktuell angepasst und modifiziert werden kann. $\mathrm{Zu}$ diesem Z weck wurden Lehr-LernArrangements in kleinere, abgeschlossene Einheiten zerlegt. Diese sind leichter zu be- 
schreiben, da sie überschaubarer sind. Außerdem lassen sie sich für neue Lehr-Lernkontexte adäquat arrangieren. D amit steht die D okumentation eines gestalteten Lehr-Lernprozesses, der mit computerbasierten Medien unterstützt wird, im Zentrum einer solchen "didaktischen Einheit". Ein Beispiel ist die Ge-staltungseinheit

\section{"Kurzbeschreibung \\ Wöchentliche Aufgaben werden zwischen den wöchentlichen Seminarsitzungen von den Studierenden bearbeitet. Die Bearbeitung wird durch die Verwendung einer internetba- sierten Groupware, z. B. BSCW, unterstützt. Auf die wöchentlichen Aufgaben erfolgt ein regelmäßiges Feedback. \\ Zielsetzungen, didaktische Motivation Vorbereitung auf die Seminarsitzung und Einführung in die Thematik sowie Rückmel- dung des Kenntnisstandes der Seminarteil- nehmerinnen und Seminarteilnehmer an die Vortragenden." \\ (Auszug aus dem Didaktischen Design Pat- tern "Wöchentliche Aufgaben") 3}

"Wöchentliche Aufgaben"2.

\section{Wahl der Beschreibungsmethode}

Welches Beschreibungsmittel eignet sich für die Dokumentation solcher wieder verwendbarer Einheiten am besten?

Interessante Anhaltspunkte bieten Beschreibungsverfahren aus dem Bereich des Software Engineerings, die von Schroeder (2002) in ersten Ansätzen für didaktische Kontexte angepasst wurden. Es hat sich in der Projektarbeit von VIB gezeigt, dass sich D esign Pattern als semiformale, textbasierte Beschreibungsform für das Ziel, "didaktisches G estaltungswissen" über die Grenzen einzelner Fächer hinaus zu kommunizieren und zu nutzen am besten eignen.

D iese Pattern Language geht auf den Architekten und Mathematiker Christopher Alexander zurück, der versuchte, ausgehend von seinen Erfahrungen und Naturbetrachtungen östlicher und asiatischer Kulturen, die Architektur in "Elementare Pattern" (Muster) aufzulösen und ihre Beziehungen untereinander festzuhalten: "Er suchte nach einer Sprache aus archetypischen Formen und Beziehungen, die den zeitlosen Architekturen von traditionellen Gemeinschaften entsprechen. D ie gefundenen Ableitungen der Pattern rühren vielfältig von Beobachtungen bewährter, beständiger und "guter" Architektur ab." 4 Sein Ziel bestand in der Dokumentation der Lösung immer wiederkehrender architektonischer Probleme: "Each pattern describes a problem which occurs over and over again in our environment, and then describes the core of the solutions to that problem, in such a way that you can use this solution a million times over, without ever doing it in the same way twice." 5 D ie von Alexander formulierten Pattern sind unter der so genannten "Pattern Language" zusammengefasst.

Die grundlegende Idee der Pattern Language bzw. die der einzelnen Pattern scheinen den Anforderungen des VIB-Projekts in allen Aspekten zu genügen:

- Im Zentrum der Beschreibungen, der D okumentation, stehen die Erfahrungen von Experten (hier: Projektmitarbeiter der Teilprojekte).

- Es findet eine Abstraktion des Expertenwissens statt, um die Wiederverwendbarkeit zu gewährleisten (Verankerung der Konzepte in der Hochschule).

D as folgende Zitat fasst alle Aspekte der Pattern präzise zusammen: "A pattern language is nothing more than a precise way of describing someone's experiences." 6

\section{Didaktische Design Pattem}

Im Sinne von Alexander stellt eine "didaktische Einheit" ein Pattern dar. Solche Didaktischen D esign Pattern werden entlang einer klaren Beschreibungsstruktur entwickelt. Dies hat zum Ziel, didaktisches Wissen und damit letztendlich didaktische Kompetenzen ohne großen Qualitätsverlust weiterzugeben. Klare Strukturen und Beschreibungsvorschriften verringern im positiven Sinne den Interpretationsspielraum und garantieren auf diese Weise, dass möglichst viel von dem "Gemeinten" auch weitergeben werden kann. Im Rahmen des VIB-Projekts wurde die bei Schroeder (2002) beschriebene Struktur weiterentwickelt und in einem "Meta-Pattern" zusammengestellt.

Die Beschreibung einer "didaktischen Einheit" umfasst

- formale Aspekte wie Name und Autor des Pattern

- inhaltliche Aspekte wie eine Kurzbeschreibung und die konkrete D urchführung

- kontextuelle Aspekte z. B. Vorschläge, wie diese Einheit in eine Hochschulveranstaltung eingebunden werden kann

- konkrete Beispiele (und Referenzen)

Kategonisienung Didaktischer Design Pattern

Neben der Erstellung einzelner Pattern muss auch eine "Sprache" gefunden werden, wie die Vielzahl entstandener Pattern für die G estaltung von computerunterstützten Lehr-Lernprozessen 


\begin{abstract}
"D urchführung
Die wöchentliche Aufgabe wird jeweils zu einem bestimmten Termin in die Groupware eingestellt. Auch die Bearbeitungsergebnisse der Studierenden haben im gemeinsamen Arbeitsbereich der Groupware ihren Ort. D er Bearbeitungszeitraum und Abgabetermin ist festgelegt und wird auch strikt kontrolliert, da die Bearbeitungen zur Vorbereitung des Seminars gebraucht werden. D as Feedback für die Bearbeitung erfolgt individuell (per Email) oder für alle Studierenden des Seminars erkennbar im Rahmen der Möglichkeiten der Groupware. D as Feedback kann durch die Lehrperson erfolgen oder durch einzelne Studierende, die sich z.B. im Rahmen eines Vortrags für ein Seminarthema speziell vorbereitet haben.

Einbindung in den Seminarkontext

Wöchentliche Aufgaben können jederzeit (durch Aushang, im WWW, in der G roupware oder per E-mail) gestellt werden. Die Rückmeldung in elektronischer Form erleichtert die Zusammenfassung und Aufbereitung für die Seminarsitzung.

Konkretes Beispiel

Beispiel aus einem Fachdidaktischen Hauptseminar im Rahmen des Lehramtsstudiums für G rund- und Hauptschulen an der Pädagogischen Hochschule Ludwigsburg.

Rahmenthema: Veranschaulichen

Aufgabenstellung:

Bearbeitungsgrundlage: http:/ / www.mste.uiuc.edu/ users/ carvell/ rectperim/ RectPerim. html

1. Beschreiben Sie zunächst, welcher Sachverhalt mit diesem Applet dargestellt wird.

2. Welche Vor- und Nachteile sehen Sie beim Einsatz dieses Applets im Unterricht.

3. Beschreiben Sie kurz eine Unterrichtssequenz, in der dieses Applet eingesetzt werden kann."
\end{abstract}

(Auszug aus dem Didaktischen D esign Pattern "Wöchentliche Aufgaben”)

Reinmann-Rothmeier \& Mandl 2000) orientiert. Diese Art der Kategorisierung der in VIB entwickelten Pattern (ca. 25) soll die Suche nach adäquaten Pattern erleichtern. Folgende Aspekte wurden berücksichtigt: Vor- und Nachbereitung von Veranstaltungen, Präsentation, Kommunikation / Kooperation, Evaluation.

Sucht eine Lehrperson eine computerunterstützte Form der Veranstaltungsvorbereitung, schaut sie unter der Kategorie Vor- und Nachbereitung von Veranstaltungen nach. Sie findet in dieser Kategorie neben Pattern wie "Vorbereitung einer Seminarsitzung mit Hilfe einer Studienumgebung und Arbeitsaufträgen per E-mail" beispielsweise das Pattern "Wöchentliche Aufgaben". In diesem Pattern wird das Vorgehen genau beschrieben, mögliche Varianten und potenzielle Problemstellen genannt. Auch Aspekte der Medienkompetenz werden - in der Kategorie "Technische Voraussetzungen" - berücksichtigt. Die Lehrperson entscheidet dann, ob diese Einheit in die von ihr geplante Veranstaltung passt. Natürlich ist im Falle eines Pattern-Einsatzes, dieses auf den eigenen Lehr-Lernkontext zu modifizieren, d.h. in unserem konkreten Fall: Es muss z.B. überlegt werden, wie häufig solche "wöchentlichen Aufgaben" gemacht werden sollen und wer das entsprechende Feedback geben soll.
Das Ziel der Didaktischen Design Pattern besteht somit darin, eine Weiterentwicklung von mediengestützten Lehr-Lemformen für Hochschulveranstaltungen anzuregen. Auf diese Weise soll die Q ualität der Lehre positiv beeinflusst und den beschriebenen mediendidaktischen Herausforderungen durch die digitalen Medien adäquat begegnet werden.

\section{Literatur}

Alexander, Ch., Ishikawa, S., Silverstein, M., Jacobson, M., Fiksdahl-King, I. \& Angel S. (1977). A Pattern Language. Towns, Buildings, Construction. New York: O xford University Press.

Reinmann-Rothmeier, G. \& Mandl, H. (2000). Individuelles Management. Strategien für den persönlichen Umgang mit Information und Wissen am Arbeitsplatz. Bern: Huber.

Reinmann-Rothmeier, G. (2002). Mediendidaktik und Wissensmanagement. In: Medienpädagogik, Heft 2, 2002. O nline: http:/ / www. medienpaed.com/ 02-2/ reinmann1.pdf [Stand: 2003-09-10].

Schroeder, U. (2002). Specification of Virtualization Processes in University Education. Online: http:/ / www-i9.informatik.rwth-aachen.de/ L uFG / forschung/ paper/ ssgrr2002w/ DidSpec| - Aquila.pdf. 


\section{Intemetquellen}

http:/ / www.uni-weimar.de/ donath/ calexander98/ ca98-html.htm [Stand: 2003-09-16]. http:/ / www.lmu.ac.uk/ ies/ comp/ research/ isle/ patterns/ background.htm [Stand: 2003-09-16].

http:/ / www-lifia.info.unlp.edu.ar/ ppp/ format. htm [Stand: 2003-08-01].

http:/ / www.vib-bw.de [Stand: 2003-09-16].

\section{Anmerkungen}

1 VIB steht für Virtualisierung im Bildungsbereich.

2 Auszüge aus dem Didaktischen D esign Pattern "Wöchentliche Aufgabe" werden hier zur Verdeutlichung der Beschreibung an unterschiedlichen Stellen eingefügt.

3 Autoren: Christine Bescherer \& Dieter Klaudt.

4 http:/ / www.uni-weimar.de/ donath/ calexander98/ ca98-html.htm [Stand: 2003-09-16].
5 http:/ / www.lmu.ac.uk/ ies/ comp/ research/ isle/ patterns/ background.htm [Stand: 2003-09-16].

6 Lambardi (2000), S.1.

7 Ein didaktisches Design Pattern beschreibt nach unseren Vorstellungen eine abgeschlossene, auch in anderen Kontexten wieder verwendbare Einheit. Sie muss hochschuldidaktische Relevanz haben, d. h. auf andere Fachdisziplinen übertragbar sein. Im Zentrum steht ein LehrLernprozess, der mit computerbasierten Medien unterstützt wird. Einzelne didaktische Pattern können zu Lehr-Lern-Arrangements (zusammengesetzte Didaktische Pattern) verbunden werden.

8 Vgl. auch O nline: http:// www-lifia.info. unlp.edu.ar/ ppp/ format.htm [Stand: 2003-0801].

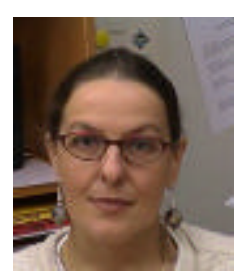

\section{Dr. Rose Vogel}

Studium der Fächer Mathematik und Biologie für das Lehramt an Realschulen; seit 1993 am Institut für Mathematik und Informatik an der Pädagogischen Hochschule Ludwigsburg; Mitarbeit im Konzeptionsbereich des Verbundprojekts "Virtualisierung im Bildungsbereich (VIB)"; Arbeits- und Forschungsschwerpunkte: Lern- und D enkstrategien in Mathematik, Sprache und Mathematik, Frauen- und Geschlechterforschung, Hochschuldidaktik.

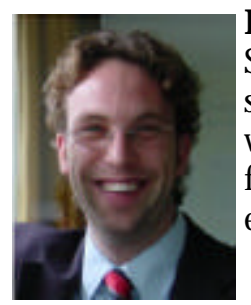

Dipl.-Päd. Sven Wippermann

Studium der Pädagogik (Schwerpunkt Medienpädagogik) an der Universität Bielefeld; seit 2001 als wissenschaftlicher Mitarbeiter an der Pädagogischen Hochschule in Ludwigsburg. Zunächst war er innerhalb des Projekts "Virtualisierung im Bildungsbereich" für den Transfer der Ergebnisse verantwortlich. Seit Mitte 2003 konzipiert und realisiert er digital unterstützte Lehr-Lern-Arrangements am Institut für Bildungsmanagement. 


\section{Neue Buchreihe}

\section{MANFRED L. PiRner}

Im Zusammenhang mit der Veröffentlichung der Tagungsdokumentation zur IZMM-Tagung 2002, "Homo medialis. Perspektiven und Probleme einer Anthropologie der Medien", wurde eine neue Buchreihe mit dem Titel "medienpädagogik intendisziplinär" ins Leben gerufen. Herausgeber sind die an der Pädagogischen Hochschule Ludwigsburg tätigen Wissenschaftler Horst Niesyto (Professor für Erziehungswissenschaft mit Schwerpunkt Medienpädagogik), Manfred L. Pirner (Professor für ev. Theologie und Religionspädagogik) und Matthias Rath (Professor für Philosophie).

A us dem Reihen-H erausgeber- $V$ orwort zum Buch "H omo medialis" :

Angesichts der Komplexität des Mediensystems in modernen $\mathrm{G}$ esellschaften und angesichts der Bedeutung, welche die Medien für nahezu alle Bereiche unseres Lebens gewonnen haben, sind Medienforschung und Medienpädagogik vorzugsweise interdisziplinär zu betreiben. In Bezug auf die Praxis der Medienerziehung und Medienbildung lassen sich inzwischen viele Reihen und Zeitschriften finden, die eine Verständigung über das gemeinsame Praxisfeld ermöglichen. Ein Disziplinen übergreifender Forschungszusammenhang jedoch verlangt verstärkt nach theoriebildender Arbeit, die erst noch im Wachsen ist. Diese Einsicht führte im Jahr 1998 zur Gründung des "Interdisziplinären Zentrums für Medienpädagogik und Medienforschung" (IZMM) an der Pädagogischen Hochschule Ludwigsburg. A uf Initiative von Horst Niesyto wurde so ein Diskussions- und Arbeitszusammenhang geschaffen, um die Kooperation zwischen interessierten Wissenschaftlerinnen und Wissenschaftlem im Bereich Medienpädagogik und Medienforschung instituts- und fakultätsübergreifend zu intensivieren.

Neben regelmäßigen Arbeitstreffen, Lehrveranstaltungsreihen, Forschungskolloquien und Gastvorträgen wurden in den vergangenen Jahren mehrere Fachtagungen durchgeführt. Ergebnisse aus diesen Forschungs- und Diskussionszusammenhängen sind teilweise bereits im kopaed-Verlag erschienen ("Selbstausdruck mit Medien. Eigenproduktion mit Medien als Gegenstand der Kindheits- und Jugendforschung", "Videoculture. Video und interkulturelle Kommunikation", beide hg. von Horst Niesyto). Außerdem wird seit zwei Jahren eine OnlineZeitschrift, die "Ludwigsburger Beiträge zur Medienpädagogik" herausgegeben, die halbjährlich erscheint (www.ph-ludwigsburg.de/ medien1/ intzent.htm).
Mit der neuen Buchreihe "medienpädagogik interdisziplinär", deren erster Band hier vorliegt, soll ein Forum geboten werden, das über die Tagungsdokumentationen und sonstigen Veröffentlichungen des IZMM hinaus auch anderen medienpädagogischen Arbeiten mit interdisziplinären Akzenten aus dem ganzen deutschen Sprachraum offen steht. Wir hoffen, dass die Vernetzung der medienpädagogischen Forschungen und Theoriebildungen dadurch weiter vorangetrieben und Medienbildung in möglichst vielfältigen schulischen und außerschulischen Kontexten verankert werden kann.

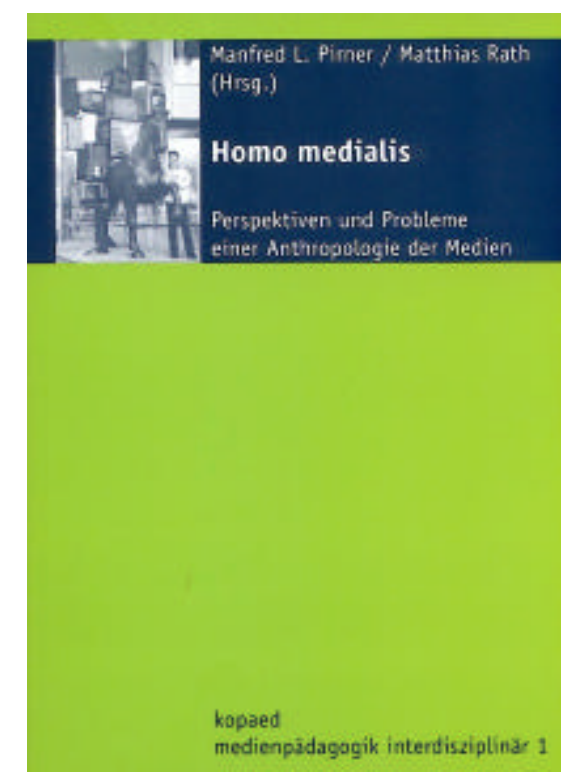




\section{Aktuelles aus der Abteilung Me- dienpädagogik}

\section{HORST NIESYTO}

\section{Archiv “Audiovisuelle Jugendkultu- ren"}

Der Aufbau eines Archivs "Audiovisuelle Jugendkulturen" findet in Zusammenhang mit der Seminar- und Forschungsarbeit von Prof. Dr. Horst Niesyto und verschiedenen Mitarbeiter/ innen statt. Die Video- und Medienproduktionen stammen vor allem aus Forschungsprojekten wie "VideoCulture" und "Chicam". Hinzu kommen kleinere Studien im Rahmen von Seminaren (Hausarbeiten) sowie Abschlussarbeiten von Studierenden, für die auch Eigenproduktionen Jugendlicher aus anderen Kontexten herangezogen werden.

Grundlage ist der Forschungsansatz "E igenproduktionen mit Medien". Zentrales Anliegen dieses Forschungsansatzes ist es,

- die Alltagspraxis von Kindern und Jugendlichen im Bereich der Mediennutzung mit medienethnographischen Methoden zu untersuchen (neben Interviews, teilnehmender Beobachtung auch Einsatz von Foto, Video und Multimedia/ Netzkommunikation);

- Kindern und Jugendlichen auch in Forschungszusammenhängen die Chance zu geben, eigene Themen, Erfahrungen, G efühle, Phantasien mittels Medien auszudrücken und hierüber Zugang zu ihrem Welterleben zu erhalten.

Ein Schwerpunkt liegt auf dem Selbstausdruck und der Kommunikation mit präsentativsymbolischen Formen (Bild, Musik, Körpersprache).

Bei dem Archiv handelt es sich um einen Präsenzbestand. Ein erster Grundstock an Eigenproduktionen wird ab Ende des Jahres 2003 in der Mediothek des Medienzentrums der PHL zugänglich sein (L 202, Frau Ebner, Tel 07141/ 140216). Langfristig ist geplant, ausgewählte Eigenproduktionen (mit Kontextinformationen) auch im Internet zugänglich zu machen.

D as Archiv enthält ausgewählte Filme, Studien und Materialien und wird schrittweise aufgebaut. Es wird folgende Teile umfassen:

- Forschungsprojekte der Abteilung Medienpädagogik

- Forschungsprojekte von anderen Einrichtungen und Personen

- Deutscher Jugendvideopreis (Kooperation mit dem KJF)

- Video- und Medienproduktionen aus weiteren Einrichtungen (D eutschland)
- Video- und Medienproduktionen (international)

- Ausgewählte Studien von Studierenden (Seminarkontexte)

- Zulassungs- und Diplomarbeiten von Studierenden.

Im WS 2002/ 03 entstand in Verbindung mit dem Seminar "Audiovisuelle Jugendkulturen Themen, Lebensgefühle, Ausdrucksformen" (Prof. Dr. Horst Niesyto) eine Kooperation zwischen der Abteilung Medienpädagogik und dem Kinder- und Jugendfilmzentrum in D eutschland, Remscheid (KJF) (www.kjf.de). Die Abteilung Medienpädagogik hat die Möglichkeit, Videofilme aus dem jährlich bundesweit stattfindenden "D eutschen Jugendvideopreis" auszuwählen und diese für Videofilmanalysen und das Archiv "Audiovisuelle Jugendkulturen" zu nutzen.

\section{"Grundlagen der Medienpädago- gik" - Kooperationsprojekt der PHen Freiburg, Ludwigsburg und Schwäbisch Gmünd}

Prof. Dr. Sven Kommer (PH Freiburg), Dipl.Päd. Wolfgang Maier (PH Schwäbisch G münd) und Prof. Dr. Horst Niesyto (PH Ludwigsburg) trafen sich am 29.09.2003 an der PHL, um ein Rahmenkonzept für eine Seminarveranstaltung "Grundlagen der Medienpädagogik" zu besprechen. Das Seminar soll im SoSe 2004 an allen drei Orten für Studierende aus verschiedenen Studiengängen angeboten werden und alle wesentlichen Inhaltsbereiche handlungsorientierter Medienpädagogik im Überblick behandeln. Ein von Horst Niesyto vorgelegtes Rahmenkonzept wird im Laufe des WS 2003/ 04 arbeitsteilig im Hinblick auf die Erarbeitung von Studienbausteinen konkretisiert werden. Vorhandene Spezialisierungen und Materialien sollen genutzt und über eine gemeinsame Kommunikationsplattform den Studierenden zugänglich gemacht werden. Die Leistungsnachweise sollen in Form von Portfolios erfolgen, die insbesondere praxisbezogene Aufgabenstellungen umfassen.

\section{Kooperation mit der PH Zürich}

Prof. Dr. Heinz Moser, Departementsleiter für den Bereich Wissensmanagement an der $\mathrm{PH}$ Zürich, und Prof. Dr. Horst Niesyto, Leiter der Abteilung Medienpädagogik an der $\mathrm{PH}$ Ludwigsburg, vereinbarten, die vorhandene Kooperation zu intensivieren. Gemeinsames Interesse ist der Austausch über aktuelle Forschungsprojekte im Bereich "Migration und Medien", über 
Inhalte und Formen einer zeitgemäßen Medienbildung im Rahmen der Lehrerbildung sowie die Reflexion des Verhältnisses der Medienpädagogik zu den einzelnen Fachwissenschaften und Fachdidaktiken. In Planung sind $u$. a. ein Austausch zwischen der Ludwigsburger Forschungsgruppe (EU-Projekt CHICAM) und der Züricher Forschungsgruppe (Forschungsprojekt beim Schweizerischen Nationalfonds im Bereich "Migration und Medien") sowie ein Treffen von Züricher Kolleginnen und Kollegen (Bereiche Wissensmanagement und Medienbildung) mit Kolleginnen und Kollegen des IZMM (Interdisziplinären Zentrums für Medienpädagogik und Medienforschung) in Ludwigsburg im Herbst 2004.

\section{Kooperation mit dem Landesme- dienzentrum Baden-Württemberg}

Hanns-Georg Helwerth, Leiter des Standorts Stuttgart des Landesmedienzentrums BadenWürttemberg, und Prof. Dr. Horst Niesyto entwickelten im Rahmen eines Informations- und Erfahrungsaustauschs verschiedene Überlegungen, die Kooperation zwischen dem LMZ und der Medienpädagogik an der PHL zu verstärken. So ist u.a. daran gedacht, im Rahmen des aktuellen Projekts "Medi@Culture" Studierenden die Möglichkeit zur Hospitation in Unterrichtsprojekten an einzelnen Schulen zu geben, verbunden mit Aufgaben zur Beobachtung und Dokumentation von Prozessabläufen. Gemeinsames Interesse ist es, ein regionales Netzwerk im Bereich Medienpädagogik voranzubringen, insbesondere was die Kooperation von schulischer und außerschulischer Medienbildung betrifft. D as LMZ erklärte sich in diesem Zusammenhang bereit, auf unterschiedlichen Ebenen an einem für das SoSe 2004 geplanten "Tag der Medienpädagogik" an der PH Ludwigsburg aktiv mitzuwirken.

\section{Vorträge und Workshop-Beteiligung von Mitarbeiter/ innen der Abteilung Medienpädagogik}

In den vergangenen Monaten beteiligten sich Mitarbeiter/ innen der Abteilung Medienpädagogik an verschiedenen Veranstaltungen mit Vorträgen und Workshop-Beiträgen:

\section{April 2003}

Beitrag von Peter Holzwarth im Rahmen der Tagung "Lebensweltorientierung in der medienpädagogischen Praxis mit Jungen und Mädchen - Erfahrungen und Ideen" in Waiblingen. (Kreisjugendring Esslingen e.V. und Akademie der Jugendarbeit Baden-Württemberg e.V.). Thema: "Eu-Projekt CHICAM - Konzept und bisherige Projektpraxis".

\section{April 2003}

Beitrag von Peter Holzwarth im Rahmen der internationalen Tagung Medienkompetenz und Medienleistungen in der Informationsgesellschaft, Universität Zürich, Institut für Publizistikwissenschaft und Medienforschung, 11.12.4.2003. Thema: "Audio-visuelle Kompetenz und interkulturelles Symbolverstehen".

\section{Mai 2003}

Horst Niesyto, Peter Holzwarth und Bjöm Maurer: Präsentation des EU-Projekts CHICAM im Rahmen des 2. Treffens des "Wissenschaftsforums Migration und Integration Baden-Württemberg" (8. Medienforum "Migranten bei uns") im SWR Funkhaus Stuttgart.

\section{Juni 2003}

Vortrag von Horst Niesyto vor dem Kuratorium des KJF (Kinder- und Jugendfilmzentrum D eutschland) in Düsseldorf. Thema: "Analyse und Verstehen von audiovisuellen Eigenproduktionen".

\section{Juni 2003}

Vortrag von Horst Niesyto auf dem internationalen AGORA-Kongress in Bologna über Children's Programming in E urope and in the Mediterranea. Thema: "Childhood, practical media work and qualitative research".

\section{Juli 2003}

Vortrag von Björn Maurer im Rahmen des Medienpädagogischen Fachtags der Stuttgarter Kinderfilmtage. Thema: "EU Forschungsprojekt CHICAM - Erfahrungen und Ergebnisse aus der Medienpraxis".

\section{September 2003}

Vortrag von Björn Maurer auf der netzkom:/ / synchronicity in Bonn (Veranstalter: JFC Köln). Thema: "Interkulturelle Medienarbeit - international und online".

\section{0 ktober 2003}

Vortrag von Horst Niesyto in der AG "Visuelle Anthropologie" im Rahmen des Jahreskongresses der D GV (Deutsche G esellschaft für Völkerkunde) in Hamburg. Thema: "Video and Global Communication".

\section{0 ktober 2003}

Workshopleitung (Horst Niesyto zusammen mit Karin D anner und Björn Maurer) zum Thema "Selbstausdruck mit neuen Medien" im Rahmen des Symposiums "Mapping Blind Spaces. Neue Wege zwischen Kunst und Bildung" (Veranstalter: IGBK, Museum für Neue Kunst / KZM, Landesakademie Schloss Rotenfels). 